\title{
Adult-Onset Still's Disease Associated with Thyroid Dysfunction: Case Report and Review of the Literature
}

\author{
Yingchun $\mathrm{Hu}^{1}$, Han Wang ${ }^{2}$ and Juelin Deng ${ }^{*}, 1$ \\ ${ }^{I}$ Department of Geriatrics, West China Hospital, Sichuan University, People's Republic of China \\ ${ }^{2}$ Department of Cardiology, The Third People's Hospital of Chengdu, Chengdu, Sichuan, People's Republic of China
}

\begin{abstract}
To our knowledge, the possible unveiled interaction between adult-onset Still's disease (AOSD) with autoimmune thyroid disease (AITD) has never been reported although it is well established that systemic autoimmune disease may usually occur in relation to AITD. As increasingly clear links of AITD with other autoimmune disease such as systemic lupus erythematosus (SLE), rheumatoid arthritis (RA), and primary Sjögren's syndrome (pSS) have been reported, and the incidence of AOSD concurrent AITD draws our attention rapidly. In this study, we searched relevant literatures published in the past 30 years to explore that condition.
\end{abstract}

Keywords: Adult-onset Still's disease, autoimmune thyroid disease.

\section{INTRODUCTION}

Adult-onset Still's disease (AOSD) is a rare systemic autoimmune syndrome of unclear etiology. In 1971, fourteen adults with arthritis and systemic symptoms in accordance with the features of juvenile rheumatoid arthritis or Still's disease were described by Bywaters. From then on, AOSD was regarded as a different disorder gradually by us [1], which was characterized by spiking fever, pruritic or nonpruritic evanescent rash, arthritis, pharyngitis, leucocytosis and lymphadenopathy, and less frequently, hepatitis and polyserositis [2]. There is no single diagnostic test for AOSD and it is mostly based on clinical and laboratory criteria with the exclusion of other autoimmune diseases, neoplasms and infection [1]. With several diagnostic criteria developed over the years, Yamaguchi's criteria has been the most widely used as a result of higher sensitivity (96.2\%) and specificity (92.1\%) [3]. Thyroid disease of autoimmune origin is a frequent condition affecting $1 \%$ to $5 \%$ of general population, especially mostly seen in women of their third to fifth decades. Autoimmune thyroid disease (AITD), usually includes Graves' disease (GD) and Hashimoto's thyroiditis (HT), which are the most common reasons for thyroid disorders. An interplay among some conditions such as immune mechanism, environmental (for example, infection, iodine and stress), genetic and constitutional factors contributes to the mechanism of AITD. GD, the most common cause of thyrotoxicosis, is more prone to a family history with thyroid disease, especially with GD. GD can be incited by the combination of autoantibodies with TSH recepor which is activated then to stimulate thyrocyte growth and function. Through regulating

*Address correspondence to this author at the Department of Geriatrics, West China Hospital, Sichuan University, 37 Guo Xue Xiang, Chengdu, Sichuan 610041, People's Republic of China; Tel: 028-85423329; E-mail: yingchunh2013@163.com against thyroid antigen, HT turns into the most prevalent reason for hypothyroidism, which gets in connection with various endocrine disorders such as type 1 diabetes mellitus, Addison's disease and hypogonadism [4]. Diagnosis of AITD is based upon clinical manifestations and persuasive laboratory results including thyroid hormone, antibodies against thyroid peroxidase (TPO) and thyroglobulin (TG). 98\% AITD are positive for either antibodies, so a negative test may rule out AITD [5].

\section{CASE}

A 43-year-old female patient without evident disease previously presented to our hospital with a 4-month history of intermittent wandering arthralgia on the left side, and also with spiking fever (the highest body temperature up to $40^{\circ} \mathrm{C}$ ), thoracalgia and sputum for 3 months. According to the examinations of the local hospital, computerized tomography (CT) scan of the chest showed bilateral pleural effusions leading to oppressive lung parenchyma; mild inflammatory infiltration of both lungs; slightly pericardial effusion; cardiomegaly and swelling lymph nodes on the right cardiodiaphragmatic angle. Distinctive laboratory findings, as shown in Table $\mathbf{1}$, easily played a role in establishing the following diagnoses: secondary pulmonary tuberculosis; tuberculous pleuritis; tuberculous pericarditis and hyperthyroidism. Therefore, the antituberculous chemotherapy and anti-thyroid drug (methimazole) were initiated; however, a poor response was obtained. Then the patient transferred to our rheumatology ward. On physical examination, she appeared irritable, general fatigue and underweight. And palpable lymph nodes at bilateral inguinal region and supraclavicular fossa were also found. Laboratory test are shown in Table 1. According to the laboratorical results of the local and our hospital, Graves' disease (GD) was diagnosed. 
Table 1. The Laboratorical results of this patient in local hospital and in our hospital.

\begin{tabular}{|c|c|c|c|c|c|c|c|c|}
\hline & Neutrophils & ESR $(\mathbf{m m} / \mathbf{h})$ & ANA and RF & C3 and C4 & $\begin{array}{c}\text { FT3, FT4, } \\
\text { TT3 and TT4 }\end{array}$ & TSH & $\begin{array}{c}\text { TGAb, TRAb } \\
\text { and TPOAb }\end{array}$ & $\begin{array}{c}\text { Routine } \\
\text { Cultures }\end{array}$ \\
\hline \hline Local hospital & -- & $70($ elevated) & Negative & Normal & Significantly elevated & decreased & -- \\
\hline Our hospital & $86.5 \%$ & -- & Negative & Normal & Normal & decreased & Moderately elevated & normal \\
\hline
\end{tabular}

FT3: free tri-iodothyronine, FT4: free thyroxine, TT3: total tri-iodothyronine, TT4: total thyroxine, TSH: thyroid-stimulating hormone, TGAb: anti-thyroglobulin antibody, TRAb: thyrotrophin receptor antibody, TPOAb: thyroid peroxidase anti-body; Routine cultures include: blood, stool, urine and throat swab, -- mean that the detailed information was not available.

Contrast-CT scan showed infection of bilateral lungs, slightly pericardial effusion and swelling lymph nodes at bilateral inguinal region and supraclavicular fossa. An abdominal ultrasound revealed splenomegaly. Thyroid ultrasonography revealed heterogeneous in echoic distribution and rich blood perfusion. Electrocardiogram showed only sinus tachycardia. A bone marrow biopsy and cytologic examination disclosed a hypercellular marrow hinting no lymphoma or other malignancies. Axillary lymph node biopsy displayed no lymphoma. According to the laboratory results, imaging studies, necessary exclusion and Yamaguchi et al. criteria, this case fulfilled the 1, 2 and 4 items of major criteria and 2 and 4 items of minor criteria, are shown in Table 2. AOSD was therefore diagnosed.

Since hospitalization in our ward, the patient had undergone an intermittent spiking fever (range from $38^{\circ} \mathrm{C}$ to $40.1^{\circ} \mathrm{C}$ ) with evanescent rash. Considering the side effect of fever induced by antituberculous drugs, so stopped the treatment. According to the thyroid hormone level mentioned above, the thyroid function was improved and temporarily anti-thyroid drugs did not appear to be used by us. Although antifebrile drugs such as antondin and radix bupleuri (a traditional medicine that enable treat fever) were injected, and temperature was kept high. But when methylprednisone $30 \mathrm{mg}$ per day was initiated, fever and rash gradually disappeared as well as thoracalgia. After she had received 2 weeks of therapy, the dose of prednisone tapered slowly. The secondary thyroid hormone test indicated remarkable hyperthyroidism (significantly increased FT3, FT4 and decreased TSH). Hence, methimazole was added. A week later, she was discharged with normal thyroid hormone and improved symptoms. CT re-examination demonstrated the disappearance of inflammation. During 1-year follow-up, the both diseases activity remained in remission.

\section{DISCUSSION}

As far as we know, the association between thyroid disorders and AOSD has never been reported. Nonetheless, the association of AITD with rheumatoid arthritis, systemic lupus erythematosus, Sjögren's syndrome and Myasthenia gravis has usually been reported [6-8]. And the systemic autoimmune disease related to AITD has been widely known. Moreover, AOSD is considered as a varirant of rheumatoid arthritis, so the similar condition of thyroid disorders with AOSD may exist. In our study, coincidental link could not be ruled out.

In this case, the examination outside our hospital showed hyperthyroidism and anti-thyroid drugs were prescribed, which led to the normal FT3, FT4, TT3 and TT4 level and decreased TSH in our hospital. Therefore, the anti-thyroid drugs were discontinued. But the spiking thyroid hormone changes occurred during hospitalization. Two explanation might be correlated to this relapse. Firstly, methimazole was stopped and the recurrence happened; secondly, AOSD and thyroid gland might interact with each other. An early report by Chen et al. [9] was similar to our case. In Chen's case, elevated thyroid hormone concentrations and a palpable goiter had not been observed prior to admission. Although hydroxychloroquine was prescribed, Graves' disease appeared on the ten-day hospitalization and during the course of the disease, the hyperthyroidism had been aggravated whenever AOSD was in active stage. When the AOSD was controlled, hyperthyroidism improved accordingly. Besides, a study from Japan [10] also demonstrated Basedow's disease was serious when AOSD was in active stage and the pathogenesis of AOSD might lead to the relapse of Basedow's disease. In the Japanese study, the author reviewed the another five literatures in which three reports disclosed the two diseases occurred at the same time, one reported AOSD was prior to Basedow's disease and the remaining one was not available. Whole of them illustrated AOSD might well have an obscured role in thyroid gland. Moreover, it appeared that when AOSD and thyroid dysfunction coexisted, both of the disorders did not always get remission from corticosteroids. Despite the uncertainty of the precise mechanism in AOSD, the two conditions may share similar pathogenesis such as immune response, environmental and genetic factors, which may

Table 2. The Yamagochi criteria for adult-onset Still's disease (AOSD).

\begin{tabular}{|c|c|c|c|}
\hline Criteria & Major Criteria & Minor Criteria & Diagnosis \\
\hline $\begin{array}{l}\text { Yamaguchi et al. } \\
\text { (sensitivity } 96.2 \% \text {; } \\
\text { specificity } 92.1 \% \text { ) }\end{array}$ & $\begin{array}{l}\text { 1. Fever } \geq 39^{\circ} \mathrm{C} \text {, lasting } \geq 1 \text { week } \\
\text { 2. Arthralgia, lasting } \geq 2 \text { weeks } \\
\text { 3. Typical rash } \\
\text { 4. Leukocytosis } \\
\left(\geq 10,000 / \mathrm{mm}^{3}\right) \\
\text { including } \geq 80 \% \text { of granulocytes }\end{array}$ & $\begin{array}{l}\text { 1. Sore throat } \\
\text { 2. Lymphadenopathy and/or } \\
\text { splenomegaly } \\
\text { 3. Liver dysfunction } \\
\text { 4. Negative RF and negative } \\
\text { ANA }\end{array}$ & $\begin{array}{l}\text { Exclusions: } \\
\text { 1. Infections (especially sepsis and infectious mononucleosis) } \\
\text { 2. Malignancies (especially malignant lymphoma) } \\
\text { 3. Rheumatic diseases (especially polyarteritis nodosa and } \\
\text { rheumatoid vasculitis with extra-articular features) } \\
\text { Classification of AOSD requires: } 5 \text { or more criteria including } \\
2 \text { or more major criteria }\end{array}$ \\
\hline
\end{tabular}


explain the interaction of both the disorders. Firstly, it has been demonstrated that type 1 helper cells (Th1)-mediated response, with elevated concentrations of tumor necrosis factor- $\alpha(\alpha-\mathrm{TNF})$, soluble TNF receptor-1, interleukin-6 (IL-6) and IL-18 [11], predominates in the immunopathogenesis of Still's disease [12]. IL-18 is regarded to occupy a vital role in the inflammatory cascade through regulating the Th1 response, and induces other cytokines such as IL-1 $\beta$, IL8 , TNF- $\alpha$ and IFN- $\gamma$ [13]. IL-1 $\beta$, which modifies thyroid epithelial tightness of human thyrocytes through altering the expression and localization of junction proteins [14], promotes the progression of a number of autoimmune diseases including rheumatic diseases, AITD, multiple sclerosis (MS) and myocarditis. IFN- $\gamma$, with the potential for expressing MHC class II molecules, especially HLA-DR molecules in the thyroid epithelial cells of AITD, is always initiated by the infection of thyroid epithelial cells or appearance of thyroid antigens. And, the class II molecules enable the persistence of thyroid disease through acting as antigen-presenting cells to activate an autoimmune response [5]. In conclusion, both share molecular mimicry on the IFN- $\gamma$ and interleukin level. Secondly, the cross reactivity of other autoantibodies with thyroid antigens or anti-thyroid autoantibodies or autorective $\mathrm{T}$ cells with other tissues and organs likely result in the concurrent diseases [15]. Thirdly, from genetically, HLA-DRB $1 * 04$ alleles correlated the two diseases [16-18].

Serositis, is a rare clinical manifestation characterized by pleuritis and pericarditis. In this case, the patient displayed polyserositis complicating cough, sputum and fever. It was extremely easy to misdiagnose the patient with pulmonary tuberculosis. After a period of anti-tuberculous treatment, the ideal result was not received. We could get a lesson from this study that AOSD affected systemic organs and tissues with wide variability, which even includes thyroid carcinoma as reported in the Inoue's study [19]. In that report, it shows that once AOSD is confirmed, we should also consider the malignancy thyroid disease such as papillary thyroid carcinoma.
So far, only a few reports have discussed the coexistence of AOSD and thyroid disorders. Literature research revealed five relevant reports (one in Chinese [20], one in French [21] and one in Japanese [10], and two in English [9, 22]). Among them (Table 3), two studies $[10,20]$ disclosed AOSD and thyroid disorders were diagnosed simultaneously; two $[9,22]$ indicated AOSD which was found before thyroid dysfunctions; one did not provide more information. In addition, only two reports provided the definite types of AITD (both were Graves' disease), but the remaining three studies had no information of detailed type of AITD and we also failed to obtain the type from the relevant antibodies level. As a result, from the three reports we only diagnosed hyperthyroidism or hypothyroidism. At present, we only believe the two diseases can interact with each other by their shared pathogenesis; however, the clear link between them fails to demonstrate the coexistence of AOSD and AITD is rarely published. With an eye to the limitation of this study, it would be better that when physicians diagnose their patients as one of the two, screen the another. Then we will have more chances to explore the correlation further and with the implementation of large-scale trials, it will be greatly advanced our understanding of the interplay and therapy.

\section{CONCLUSION}

In conclusion, coexistence of AOSD and AITD is so rarely published that the explicit relationship is hard to acquire, but we can believe they could interact with each other through their common pathogenesis. As more cases are reported, the hidden assiciation will become clear. Polyserositis is very scarce in the manifestation of AOSD. When, we, physicians encounter this sign, it becomes necessary to screen the relevant laboratory and imaging abnormalities. Circumstances permitting, a thyroid gland screening is recommended, especially in female patients. Finally, it is reasonable to reconsider the various manifestations of AOSD.

Table 3. Characteristic of patients with AOSD and TD.

\begin{tabular}{|c|c|c|c|c|c|c|c|c|}
\hline Ref. & Sex & Comorbidities & Age & Order of the Occurrence & Laboratory Test & Treatment & Symptoms & Prognosis \\
\hline [8] & $\mathrm{F}$ & $\begin{array}{l}\text { Graves' } \\
\text { disease }\end{array}$ & $37 y$ & AOSD prior to TD & $\begin{array}{c}\text { Elevated FT3, } \\
\text { FT4, TGAb; } \\
\text { decreased } \\
\text { TSH, TMAb(+) }\end{array}$ & $\begin{array}{c}\text { Cs, PTU, } \\
\text { propranolol, MMI }\end{array}$ & $\begin{array}{l}\text { Intermittent } \\
\text { palpitations, } \\
\text { sweating, } \\
\text { heat intolerance, } \\
\text { tremor }\end{array}$ & Recovery \\
\hline [9] & $\mathrm{F}$ & $\begin{array}{l}\text { Basedow's } \\
\text { disease }\end{array}$ & $50 \mathrm{y}$ & $\begin{array}{l}\text { the diagnosis of TD prior } \\
\text { to AOSD, but relapse } \\
\text { concurrent AOSD }\end{array}$ & $\begin{array}{l}\text { decreased TSH, } \\
\text { elevated FT4 }\end{array}$ & Cs & NA & Recovery \\
\hline [21] & $\mathrm{F}$ & hypothyroidism & $36 y$ & AOSD prior to TD & $\begin{array}{c}\text { decreased FT3, } \\
\text { FT4, TSH }\end{array}$ & Cs, MTX, Lev & $\begin{array}{l}\text { fatigue, } \\
\text { bradycardia }\end{array}$ & Recovery \\
\hline [20] & NA & hyperthyroidism & NA & NA & $\begin{array}{c}\text { elevated T4, } \\
\text { decreased TSH }\end{array}$ & NA & NA & NA \\
\hline [19] & $\mathrm{F}$ & $\begin{array}{l}\text { Hyperthyroidism and } \\
\text { hyperthyroidism crisis }\end{array}$ & $45 y$ & AOSD parallel to TD & $\begin{array}{c}\text { elevated FT3, } \\
\text { FT4; } \\
\text { decreased TSH }\end{array}$ & Cs, PTU, ISA & irritable & Recovery \\
\hline
\end{tabular}

F: female, AOSD: adult-onset Still's disease, TD: thyroid disease, FT3: free tri-iodothyronine, FT4: free thyroxine, T4: thyroxine, TSH: thyroid-stimulating hormone, TGAb: antithyroglobulin antibody, TMAb: antimicrosomal antibody, Cs: corticosteroids, PTU: propylthiouracil, MMI: methimazole, MTX: methotrexate, Lev: levothyroxine, ISA: immunosuppressive agents, NA: not available. 


\section{AUTHORS' CONTRIBUTIONS}

Juelin Deng - conduct of study, analysis and interpretation of data, critical revision/drafting of the manuscript, final approval to submit.

Han Wang - analysis and interpretation, critical revision/drafting of the manuscript, final approval to submit.

Yingchun $\mathrm{Hu}-$ analysis and interpretation of data, critical revision/drafting of the manuscript, final approval to submit.

\section{CONFLICT OF INTEREST}

The authors declare no conflict of interest. The authors alone response for the contents and writing of the paper.

\section{ACKNOWLEDGMENTS}

Declared none.

\section{REFERENCES}

[1] Bywaters EGL. Still's disease in the adult. Ann Rheum Dis 1971; 30: 121-33.

[2] Kontzias A, Efthimiou P. Adult-onset Still's disease pathogenesis, clinical manifestations and therapeutic advances. Drugs 2008; 68: 319-37.

[3] Yamaguchi M, Ohta A, Tsunematsu T, et al. Preliminary criteria for classification of adult Still's disease. J Rheumatol 1992; 19(3): 424-30.

[4] Huber A, Menconi F, Corathers S, Jacobson EM, Tomer Y. Joint genetic susceptibility to type 1 diabetes and autoimmune thyroiditis: from epidemiology to mechanisms. Endocr Rev 2008; 29(6): 697-725.

[5] Tomer Y. Mechanisms of autoimmune thyroid diseases: from genetics to epigenetics. Ann Rev Pathol 2014; 9(1): 147-56.

[6] Mihailova R, Grigorova R, Vassileva B, et al. Autoimmune thyroid disorders in juvenile chronic arthritis and systemic lupus erythematosus. Adv Exp Med Biol 1999; 445: 55-61.

[7] Harel L, Prais D, Uziel Y, et al. Increased prevalence of antithyroid antibodies and subclinical hypothyroidism in children with juvenile idiopathic arthritis. J Rheumatol 2006; 33: 164-6.
[8] Stagi S, Giani T, Simonini G, Falcini F. Thyroid function, autoimmune thyroiditis and celiac disease in juvenile idiopathic arthritis. Rheumatology 2005; 44: 517-20.

[9] Chen HS, Yu KH, Ho HH. Coexistence of adult-onset Still's Disease and autoimmune hyperthyroidism in a patient who responded to corticosteroids and $\beta$-blocker. Immunopharmacol Immunotoxicol 2010; 32(4): 696-9.

[10] Torigoe M, Miyamura T, Nakamura M, et al. Simultaneous relapse of Basedow's disease in a patient with adult-onset Still's disease (in Japanese). Jpn J Clin Immunol 2011; $34:$ 426-30.

[11] Fujii T, Nojima T, Yasuoka $\mathrm{H}$, et al. Cytokine and immunogenetic profiles in Japanese patients with adult Still's Disease. Association with Chronic articular disease. Rheumatology 2001; 40: 1398-404.

[12] Chen DY, Lan JL, Lin FJ, Hsieh TY, Wen MC. Predominance of Th1 cytokine in peripheral blood and pathological tissues of patients with active untreated adult onset Still's Disease. Ann Rheumatol 2004; 63: 1300-6.

[13] Sugiura T, Kawaguchi Y, Harigai M, et al. Association between adult-onset Still's disease and interleukin-18 gene polymorphisms. Genes Immun 2002; 3: 394-9.

[14] Zhao RJ, Zhou HY, Su SB. A critical role of interleukin-1 $\beta$ in the progression of autoimmune disease. Int Immunopharmacol 2013; 17: 658-69.

[15] Paul S, Li L, Kalaga R, et al. Characterization of thyroglobulin directed and polyreactive catalytic antibodies in autoimmune disease. J Immunol 1997; 159(3): 1530-6.

[16] Selva-O'Callaghan A, Redondo-Benito A, Trallero-Araguas E, Martinez-Gomez X, Palou E, Vilardell-Tarres M. Clinical significance of thyroid disease in patients with inflammatory myopathy. Medicine (Baltimore) 2007; 86: 293-8.

[17] Efthimiou P, Paik PK, Bielory L. Diagnosis and management of adult onset Still's Disease. Ann Rheum Dis 2006; 65: 564-72.

[18] Joung CI, Lee HS, Lee SW, et al. Association between HLA-DRB1 and clinical features of adult onset Still's Disease in Korea. Clin Exp Rheumatol 2003; 21: 489-92.

[19] Inoue R, Kato T, Kim F, et al. A case of adult-onset Still's disease (AOSD)-like manifestations abruptly during confirmation of a diagnosis of metastatic papillary thyroid carcinoma. Mod Rheumatol 2012; 22: 796-800.

[20] Fang LW, Wu YQ. Adult-onset Still's Disease complicated by hyperthyroidism crisis. New Medicine 2011; 42(9): 620-1.

[21] Kaltenbach G, Lujic J, Arpin-Bott MP, Imler M. Adult onset Still's disease and subacute thyroiditis. Rev Med Interne 1996; 17(5): 427-31

[22] Nagai Y. Adult-onset Still's Disease complicated by multiple cranial nerve palsy and hypothyroidism. J Dermatol 2008; 35: 2489.

(C) Hu et al.; Licensee Bentham Open.

This is an open access article licensed under the terms of the Creative Commons Attribution Non-Commercial License (http://creativecommons.org/licenses/by-nc/ $3.0 /$ ) which permits unrestricted, non-commercial use, distribution and reproduction in any medium, provided the work is properly cited. 